\title{
MINIMAL NUMBER OF PERIODIC POINTS \\ FOR SMOOTH SELF-MAPS \\ OF TWO-HOLED 3-DIMENSIONAL CLOSED BALL
}

\author{
Grzegorz GrafF
}

\begin{abstract}
Let $M$ be two-holed 3-dimensional closed ball, $r$ a given natural number. We consider $f$, a continuous self-map of $M$ with real eigenvalues on the second homology group, and determine the minimal number of $r$-periodic points for all smooth maps homotopic to $f$.
\end{abstract}

\section{Introduction}

Let $M$ be a compact manifold of dimension $m, r>1$ be a fixed natural number. One of the central questions in the periodic point theory concerns finding minimal number of $r$-periodic points in the homotopy class of a given continuous self-map $f$ of $M$. This problem, for manifolds of dimension not less than 3, was solved by J. Jezierski, who proved in [10] that the answer is given by the classical invariant $N F_{r}(f)$ defined by B. Jiang (cf. [11]), i.e.

$$
N F_{r}(f)=\min \left\{\# \operatorname{Fix}\left(g^{r}\right): g \sim f\right\} .
$$

However, if we consider smooth $f$ and its smooth homotopy class, the minimum in the formula (1.1) turned out to be completely different and for simplyconnected manifold it is given by the invariant $D_{r}^{m}[f]$ defined in [5]. In fact,

2000 Mathematics Subject Classification. Primary 37C25, 55M20; Secondary 37C05.

Key words and phrases. Least number of periodic points, Nielsen number, fixed point index, smooth maps.

Research supported by KBN grant No 1/P03A/03929. 
the invariant may be applied in a bit more general situation. Instead of taking a smooth $f$ and smooth homotopies, we may consider continuous $f$ and search for the minimum over smooth $g$ in the continuous homotopy class. As every smooth homotopy may be approximated by a continuous one, both approaches in the common domain lead to the same result, thus $D_{r}^{m}[f]=\min \left\{\# \operatorname{Fix}\left(g^{r}\right)\right.$ : $g \sim f$ and $g$ is smooth .

The important problem is to determine the value of $D_{r}^{m}[f]$ for different manifolds. The construction of this invariant bases on the notion of so-called $\mathrm{DD}^{m}(p)$ sequence, which is a sequence of integers which can be realized as sequence of indices at an isolated $p$-orbit for some smooth map. The minimal decomposition of the sequence of Lefschetz numbers $\left\{L\left(f^{n}\right)\right\}_{n \mid r}$ into the sum of DD ${ }^{m}(p)$ sequences, in which the contribution of each $\operatorname{DD}^{m}(p)$ sequence is equal to $p$, gives the value of $D_{r}^{m}[f]$. As, up to now, only the forms of $\operatorname{DD}^{3}(p)$-sequences are known [7], the calculations of $D_{r}^{m}[f]$ are possible for 3-dimensional manifolds. The complete description of $D_{r}^{3}[f]$ for self-maps of $S^{2} \times I$ and $S^{3}$ were given in [5] and [6], respectively.

The aim of this paper is to determine $D_{r}^{3}[f]$ for self-maps of $M$, a two-holed 3-dimensional closed ball, with real eigenvalues on the second homology group and without periodic points on the boundary.

In order to find the value of $D_{r}^{3}[f]$ we have to write down Lefschetz numbers of iterations in the form of so-called periodic expansion. It is usually difficult to obtain general form of such representation for any self-map of a given manifold. The limitation of our study to the maps with real eigenvalues results from the fact that, up to now, only for this class of maps we know the exact form of the periodic expansion.

The article is based on [5], in which the invariant $D_{r}^{m}[f]$ was introduced, and [9] where the periodic expansions of Lefschetz numbers for self-maps of $M$ were given. The case of odd $r$ is relatively easy to deal with, by the combination of the previously known theorems. Considering the case of even $r$ we notice (Theorem 5.3) that the same technics as for $S^{2} \times I$ may be applied for some self-maps of 3-dimensional manifolds with fast grow of Lefschetz numbers, which gives the common value of $D_{r}^{3}[f]$ for them. Next, we show that the considered self-maps of $M$ belong to that class of maps. The final results of the paper are given in Proposition 5.1 and Theorems 5.2 and 5.4.

\section{Periodic expansions, $\mathrm{DD}^{m}$ sequences and the definition of $D_{r}^{m}[f]$}

A sequence of indices of iterations at an isolated fixed or periodic point is an important tool used to find minimal number of periodic points in homotopy class. Let $U$ be an open subset of $\mathbb{R}^{m}$. For a map $f$ such that $f: U \rightarrow \mathbb{R}^{m}$ we 
consider $x_{0}$, an isolated fixed point for each iteration of $f$. Under this assumption the sequence of local fixed point indices $\left\{\operatorname{ind}\left(f^{n}, x_{0}\right)\right\}_{n=1}^{\infty}$ is well-defined.

Definition 2.1. For a given $d$ we define the basic sequence:

$$
\operatorname{reg}_{d}(n)= \begin{cases}d & \text { if } d \mid n \\ 0 & \text { if } d \not n\end{cases}
$$

Each $\operatorname{reg}_{d}$ is an elementary periodic sequence of the form:

$$
(0, \ldots, 0, d, 0, \ldots, 0, d, \ldots)
$$

where the non-zero entries appear for indices divisible by $d$.

Definition 2.2. For a given sequence of indices $\left\{\operatorname{ind}\left(f^{n}, x_{0}\right)\right\}_{n=1}^{\infty}$, we define the sequence of Dold coefficients $\left\{a_{d}\right\}_{d=1}^{\infty}$ by the formula:

$$
a_{d}=\frac{1}{d} \sum_{k \mid d} \mu(k) \operatorname{ind}\left(f^{(d / k)}, x_{0}\right),
$$

where $\mu$ is the Möbius function, i.e. $\mu: \mathbb{N} \rightarrow \mathbb{Z}$ is defined by the following three properties:

(a) $\mu(1)=1$,

(b) $\mu(k)=(-1)^{s}$ if $k$ is a product of $s$ different primes,

(c) $\mu(k)=0$ otherwise.

By a use of basic sequences and Dold coefficients we may write down sequences of indices of iterations in the following form, called periodic expansion (cf. [13]):

$$
\operatorname{ind}\left(f^{n}, x_{0}\right)=\sum_{d=1}^{\infty} a_{d} \operatorname{reg}_{d}(n) .
$$

Remark 2.3. A. Dold found in 1983 the following congruences called Dold relations [4]:

$$
\sum_{k \mid d} \mu(k) \operatorname{ind}\left(f^{(d / k)}, x_{0}\right) \equiv 0 \quad(\bmod d) .
$$

This implies that the sequence of Dold coefficients consists of integer numbers, and thus all coefficients $a_{d}$ in the periodic expansion (2.1) are integers.

The notion of Differential Dold sequence (DD-sequences in short) was introduced in [5] in order to define the invariant $D_{r}^{m}[f]$ (mentioned in the introduction). Briefly speaking it is a sequence which can be locally realized as a sequence of indices on an isolated $p$-orbit for some smooth map. 
Definition 2.4. A sequence of integers $\left\{c_{n}\right\}_{n=1}^{\infty}$ is called a $\operatorname{DD}^{m}(p)$-sequence if there is an isolated $p$-orbit $P$, its neighbourhood $U \subset \mathbb{R}^{m}$ and a $C^{1}$ map $\phi: U \rightarrow \mathbb{R}^{m}$ such that $c_{n}=\operatorname{ind}\left(\phi^{n}, P\right)$. If this equality holds for $n \mid r$, where $r$ is fixed, then the finite sequence $\left\{c_{n}\right\}_{n \mid r}$ will be called $\operatorname{DD}^{m}(p \mid r)$ sequence.

Using Definition 2.4 we may define the invariant $D_{r}^{m}[f]$.

Definition 2.5. Let $\left\{L\left(f^{n}\right)\right\}_{n \mid r}$ be a finite sequence of Lefschetz numbers. We decompose $\left\{L\left(f^{n}\right)\right\}_{n \mid r}$ into the sum:

$$
L\left(f^{n}\right)=c_{1}(n)+\ldots+c_{s}(n),
$$

where $c_{i}$ is a $\operatorname{DD}^{m}\left(l_{i} \mid r\right)$ sequence for $i=1, \ldots, s$. Each such decomposition determines the number $l=l_{1}+\ldots+l_{s}$. We define the number $D_{r}^{m}[f]$ as the smallest $l$ which can be obtained in this way.

The invariant $D_{r}^{m}[f]$ gives the value of the minimal number of $r$-periodic points for all smooth maps in homotopy class of $f$.

THEOREM 2.6 ([5]). Let $M$ be a smooth compact connected and simplyconnected manifold of dimension $m \geq 3$ and $r \in \mathbb{N}$ a fixed number. For $M$ with nonempty boundary we assume additionally that $f$ has no periodic points on the boundary. Then,

$$
D_{r}^{m}[f]=\min \left\{\# \operatorname{Fix}\left(g^{r}\right): g \text { is } C^{1} \text { and is homotopic to } f\right\} \text {. }
$$

In order to find the invariant $D_{r}^{m}[f]$ we must know, by Definition 2.5, the forms of all $\mathrm{DD}^{m}(p)$ sequences. It turns out that it is enough to know only the forms of $\mathrm{DD}^{m}(1)$ sequences, as the every $\mathrm{DD}^{m}(p)$ sequences can be obtained from some $\mathrm{DD}^{m}(1)$ one.

Definition 2.7. We will say that the $\operatorname{DD}^{m}(p)$ sequence $\left\{\widetilde{c_{n}}\right\}_{n}$ comes from the given $\operatorname{DD}^{m}(1)$ sequence $\left\{c_{n}\right\}_{n}$ with the periodic expansion

$$
c_{n}=\sum_{d=1}^{\infty} a_{d} \operatorname{reg}_{d}(n)
$$

if the periodic expansion of $\left\{\widetilde{c_{n}}\right\}_{n}$ has the form:

$$
\widetilde{c_{n}}=\sum_{d=1}^{\infty} a_{d} \operatorname{reg}_{p d}(n) .
$$

LEMMA 2.8 ([5, Remark 2.10]). Each $\mathrm{DD}^{m}(p)$ sequence comes from some $\mathrm{DD}^{m}(1)$ sequence. 


\section{Invariant $D_{r}^{m}$ for $m=3$}

We start our analysis with the right-hand side of the formula (2.2). Due to Lemma 2.8, we will be able to calculate $D_{r}^{3}[f]$ if we know all the forms of $\operatorname{DD}^{3}(1)$ sequences. The description of $\operatorname{DD}^{3}(1)$ sequences was given in [7] by the following theorem:

THEOREM 3.1. There are seven kinds of $\mathrm{DD}^{3}(1)$ sequences:
(A) $c_{A}(n)=a_{1} \operatorname{reg}_{1}(n)+a_{2} \operatorname{reg}_{2}(n)$,
(B) $c_{B}(n)=\operatorname{reg}_{1}(n)+a_{d} \operatorname{reg}_{d}(n)$,
(C) $c_{C}(n)=-\operatorname{reg}_{1}(n)+a_{d} \operatorname{reg}_{d}(n)$,
(D) $c_{D}(n)=a_{d} \operatorname{reg}_{d}(n)$,
(E) $c_{E}(n)=\operatorname{reg}_{1}(n)-\operatorname{reg}_{2}(n)+a_{d} \operatorname{reg}_{d}(n)$,
(F) $c_{F}(n)=\operatorname{reg}_{1}(n)+a_{d} \operatorname{reg}_{d}(n)+a_{2 d} \operatorname{reg}_{2 d}(n)$, where $d$ is odd,
(G) $c_{G}(n)=\operatorname{reg}_{1}(n)-\operatorname{reg}_{2}(n)+a_{d} \operatorname{reg}_{d}(n)+a_{2 d} \operatorname{reg}_{2 d}(n)$, where $d$ is odd.

In all cases $d \geq 3$ and $a_{i} \in \mathbb{Z}$.

What is more, in dimension 3 except for the $\operatorname{DD}^{m}(1 \mid r)$ sequences we need only some $\mathrm{DD}^{m}(2 \mid r)$ for calculating $D_{r}^{3}[f]$.

LEMMA $3.2([5])$. Let $f: M \rightarrow M$ be a $C^{1}$ map, $\operatorname{dim} M=3$, then in the definition of $D_{r}^{3}[f]$ it is enough to consider only $\mathrm{DD}^{3}(1 \mid r)$ sequences i.e. sequences which, for $n \mid r$, are of the forms $(\mathrm{A})-(\mathrm{G})$; and $\mathrm{DD}^{3}(2 \mid r)$ sequences which comes (in the sense of Definition 2.7) from the sequences of the form $(\mathrm{E})-(\mathrm{G})$.

Now, we turn to the left-hand side of the formula (2.2). By Definition 2.5 the value of $D_{r}^{m}[f]$ depends on the decomposition of Lefschetz numbers into $\operatorname{DD}^{m}(p \mid r)$ sequences. The most convenient way to study such decompositions is to find periodic expansion of Lefschetz numbers. Then, in the formula (2.2) both sides have the form of periodic expansion and the problem of minimal realization becomes a combinatorial question.

Dold congruences are valid not only for local, but also for global indices, so in the periodic expansion of Lefschetz numbers of iterations

$$
L\left(f^{n}\right)=\sum_{d=1}^{\infty} b_{d} \operatorname{reg}_{d}(n),
$$

all coefficients $b_{d}=(1 / d) \sum_{k \mid d} \mu(k) L\left(f^{d / k}\right)$ are integers.

Let $r$ be a fixed natural number. Now we introduce the following notation. By the set of algebraic period of $f$ we will call the subset of natural numbers $B(f)$, given by:

$$
B(f)=\left\{d \in \mathbb{N}: b_{d} \neq 0\right\}
$$


we will also consider its subset $B_{r}(f)$, defined as:

$$
B_{r}(f)=\left\{d \in \mathbb{N}: d \mid r \text { and } b_{d} \neq 0\right\} .
$$

Because $\mathrm{DD}^{3}(1)$ sequences have very special forms, it will be useful for us to write some first terms in the formula (3.1) (for $n \mid r$ ) separately:

$$
L\left(f^{n}\right)=b_{1} \operatorname{reg}_{1}(n)+b_{2} \operatorname{reg}_{2}(n)+b_{4} \operatorname{reg}_{4}(n)+\sum_{d \in G} b_{d} \operatorname{reg}_{d}(n),
$$

where $b_{1}, b_{2}, b_{4}$ are arbitrary integers,

$$
G=\left\{d \in \mathbb{N}: d \notin\{1,2,4\} \text { and } b_{d} \neq 0\right\}=B_{r}(f) \backslash\{1,2,4\} .
$$

Let us define $H$, the subset of $G$ :

$$
H=\left\{d \in G: d \text { is odd and } b_{d} \neq 0, b_{2 d} \neq 0\right\} .
$$

Applying the above notation we may formulate the theorem from [5], which enables us to find or estimate $D_{r}^{3}[f]$.

THEOREM 3.3.

(*) If $r$ is odd, then:

$$
D_{r}^{3}[f]= \begin{cases}\# G & \text { if }|L(f)| \leq \# G, \\ \# G+1 & \text { otherwise. }\end{cases}
$$

(**) If $r$ is even and $r \geq 4$, then:

$$
D_{r}^{3}[f] \in[\# G-\# H, \# G-\# H+2] .
$$

\section{Algebraic periods}

In this section we describe the form of periodic expansion of Lefschetz numbers for considered self-maps of a two-holed 3-dimensional closed ball. More precisely, we give the exact form of the set of algebraic periods of $f$ (cf. the formula (3.2)). We base on the description of algebraic periods for self-maps of some types of manifolds, which was given in [9].

We use homology with the coefficients in the field of rational numbers $\mathbb{Q}$. Let us consider a manifold $M$ which satisfies the assumptions of Theorem 2.6 and has non-empty boundary. We get: $H_{0}(M)=\mathbb{Q}(M$ is connected $), H_{1}(M)=0$ ( $M$ is simple-connected), $H_{2}(M)=\mathbb{Q}^{i}, H_{3}(M)=0$ ( $M$ has boundary). This is equivalent to the statement that the boundary of $M$ consists of $i+1$ pairwise disjoined 2-spheres (cf. [8]). We consider here the case $i=2$, so the boundary consists of three 2-spheres. Gluing 3-disks along these spheres we get a compact simply-connected 3-manifold, thus $S^{3}$ by Poincaré Theorem. As a result, $M$ is a closed 3-dimensional ball with two open ball removed. 
Let $f_{* 2}: H_{2}(M ; \mathbb{Q}) \rightarrow H_{2}(M ; \mathbb{Q})$, we denote by $t$ and $d$ the trace and determinant of the corresponding $2 \times 2$ integral matrix.

Theorem 4.1 cited below was originally proved in [9] for a different class of manifolds, namely for manifolds $\widetilde{M}$ with the homology $H_{0}(\widetilde{M} ; \mathbb{Q})=\mathbb{Q}$, $H_{1}(\widetilde{M} ; \mathbb{Q})=\mathbb{Q} \times \mathbb{Q}, H_{k}(\widetilde{M} ; \mathbb{Q})=0$ for $k \neq 1,2$. Nevertheless, the description of the set of algebraic periods for self-maps of $\widetilde{M}$ and $M$ is the same (except for a slight difference in the case $(\mathrm{d}))$. Assume that $\lambda_{1}, \lambda_{2}$, the eigenvalues of $f_{* 2}: H_{2}(M ; \mathbb{Q}) \rightarrow H_{2}(M ; \mathbb{Q})$, are equal to the eigenvalues of $\widetilde{f}_{* 1}: H_{1}(\widetilde{M} ; \mathbb{Q}) \rightarrow$ $H_{1}(\widetilde{M} ; \mathbb{Q})$. Then the coefficients of periodic expansion of Lefschetz numbers for self-maps of $\widetilde{M}$ are equal to

$$
\widetilde{b}_{d}=\frac{1}{d} \sum_{k \mid d} \mu(k)\left[1-\left(\lambda_{1}^{d / k}+\lambda_{2}^{d / k}\right)\right],
$$

while for $M$ we have:

$$
b_{d}=\frac{1}{d} \sum_{k \mid d} \mu(k)\left[1+\left(\lambda_{1}^{d / k}+\lambda_{2}^{d / k}\right)\right] .
$$

As $\sum_{k \mid d} \mu(k)=0$ for $k>1, \widetilde{b}_{d} \neq 0$ if and only if $b_{d} \neq 0$ for $d \neq 1$.

Theorem 4.1 ([9, Theorem B] $)$. Assume that the eigenvalues of $f_{* 2}$ are real. Let $b_{n}$ denote the nth coefficient of the periodic expansion (3.1), then the following statements hold:

(a) If $n>1$ is odd, then $b_{n}=0$ if and only if $(t, d) \in E_{o}$, where $E_{o}=$ $\{( \pm 1,0),( \pm 2,1)\} \cup\{(0, d): d \in \mathbb{Z}$ and $d \leq 0\}$.

(b) If $n>2$ is even, then $b_{n}=0$ if and only if $(t, d) \in E_{e}$, where $E_{e}=$ $\{( \pm 1,0),( \pm 2,1),(0,0),(0,-1)\}$.

(c) $b_{2}=0$ if and only if $(t, d) \in\{(1,0),(2,1),(0,0)\}$.

(d) $b_{1}=0$ if and only if $t=-1$.

\section{5. $D_{r}^{3}[f]$ for self-maps of two-holed 3-dimensional closed ball}

We begin our analysis with the special cases of the pairs $(t, d)$ which are in the sets $E_{o}$ or $E_{e}$.

Proposition 5.1. Let $r$ be odd. Then $(t, d) \in E_{o} \backslash\{(-1,0)\}$ implies $D_{r}^{3}[f]=$ $1 ;(t, d)=(-1,0)$ implies $D_{r}^{3}[f]=0$. Let $r$ be even and $(t, d) \in E_{e}$, then $D_{r}^{3}[f]=1$.

Proof. By Theorem 4.1 it is easy to observe that for odd $r$ and $(t, d) \in$ $E_{o} \backslash\{(-1,0)\}$, as well as for even $r$ and $(t, d) \in E_{e}$, we have: $L\left(f^{n}\right)=b_{1} \operatorname{reg}_{1}(n)+$ $b_{2} \operatorname{reg}_{2}(n)$ with some coefficients $b_{1}$ and $b_{2}$ which are not both equal to zero. As a consequence, the sequence of Lefschetz numbers can be realized by one sequence 
of the type (A) from Theorem 3.1. If $r$ is odd and $(t, d)=(-1,0)$, then $L\left(f^{n}\right)$ is constantly equal to zero, and thus $D_{r}^{3}[f]=0$.

Let $\zeta(r)$ denote the number of all divisors of $r$.

TheOREM 5.2. If $r$ is odd and $(t, d) \notin E_{o}$ then:

$$
D_{r}^{3}[f]= \begin{cases}\zeta(r)-1 & \text { if }-\zeta(r) \leq t \leq \zeta(r)-2, \\ \zeta(r) & \text { otherwise. }\end{cases}
$$

Proof. By Theorem 4.1(a) each $n$ dividing $r$ is an algebraic period. As a consequence, $B_{r}(f)=\{n \in \mathbb{N}: n \mid r\}$, or equivalently \# $B_{r}(f)=\zeta(r)$. Because for odd $r, G=B_{r}(f) \backslash\{1\}$, we get that

$$
\# G=\zeta(r)-1 .
$$

On the other hand, notice that $L(f)=1+t$. Now, the application of Theorem 3.3 gives the thesis.

Let us consider the case of $r$ even. First, we determine $D_{r}^{3}[f]$ in more general setting, namely for some class of self-maps of 3-manifolds. This will give the common value of $D_{r}^{3}[f]$ for the whole class, which consists of maps with positive coefficients $b_{2}$ and $b_{4}$ and such that the set of algebraic periods is equal to all natural numbers. By $\eta(r)$ we will denote the number of all odd divisors of $r$.

THEOREM 5.3. Assume that $r$ is even and $f$ is a self-map of a compact connected and simply-connected 3-manifold such that two following conditions are satisfied:

(i) $B(f)=\mathbb{N}$,

(ii) $b_{2}>0$ and $b_{4}>0$.

Then

$$
D_{r}^{3}[f]=\zeta(r)-\eta(r) .
$$

Proof. We start with the case $4 \mid r$. By Theorem $3.3(* *)$

$$
D_{r}^{3}[f] \in[\# G-\# H, \# G-\# H+2] .
$$

By Lemma 3.2 during the calculation of $D_{r}^{3}[f]$ we need the $\mathrm{DD}^{3}(1)$-sequences (A) $-(\mathrm{G})$ of Theorem 3.1 and $\mathrm{DD}^{3}(2 \mid r)$ sequences which comes from the sequences of the form (E)-(G). Applying the same argument as in the proof of Theorem 4.23 in [5] we get that for maps satisfying (i) we may use only $\mathrm{DD}^{3}(1)$-sequences (A) $-(\mathrm{G})$ of Theorem 3.1.

Now let us notice that to get the decomposition of Lefschetz numbers in the formula (3.3) for $d \neq 1,2,4$ we need at least $\# H$ sequences $(\mathrm{F})$ and $(\mathrm{G})$, each of which reduces two basic sequence $b_{d} \mathrm{reg}_{d}$ and $\# G-2 \# H$ sequences (B)-(E), each of which reduces one sequence $b_{d} \mathrm{reg}_{d}$. Because by (ii) both $b_{2}$ and $b_{4}$ are 
positive, we cannot obtain $b_{2} \mathrm{reg}_{2}$ and $b_{4} \mathrm{reg}_{4}$ as a part of the previously used sequences, and thus we need two more sequences (among them one of the type (A) in Theorem 3.1) to realize the expression

$$
b_{1} \operatorname{reg}_{1}(n)+b_{2} \operatorname{reg}_{2}(n)+b_{4} \operatorname{reg}_{4}(n) .
$$

Finally, $D_{r}^{3}[f]$ is not less than $(\# H)+(\# G-2 \# H)+2=\# G-\# H+2$, but by (5.1) this is its exact value.

Now by the assumption (i) $B_{r}(f)=\{n \in \mathbb{N}: n \mid r\}$, so \# $B_{r}(f)=\zeta(r)$. On the other hand $G=B_{r}(f) \backslash\{1,2,4\}$, and thus $\# G=\zeta(r)-3$. By Lemma 4.19 in [5], $\# H=\eta(r)-1$. Taking into account the values of $\# G$ and $\# H$ we get that:

$$
D_{r}^{3}[f]=\zeta(r)-\eta(r)
$$

The proof of the case $4 \nmid r$ may be easily obtained from the above considerations as a special case.

Theorem 5.4. Assume that $r$ is even and $(t, d) \notin E_{e}$. Then

$$
D_{r}^{3}[f]=\zeta(r)-\eta(r)
$$

Proof. By Theorem 5.3 it is enough to check whether the conditions (i) and (ii) are satisfied.

By Theorem 4.1(b), the condition (i) in Theorem 5.3 is satisfied. Now we verify the condition (ii) of Theorem 5.3. In the case of real eigenvalues $\lambda_{1}, \lambda_{2}$ of $f_{* 2}$ we have the inequality $t^{2}-4 d \geq 0$ (remind that $\left.\lambda_{1}+\lambda_{2}=t, \lambda_{1} \lambda_{2}=d\right)$. This implies that

$$
b_{2}=\frac{1}{2}\left(L\left(f^{2}\right)-L(f)\right)=\frac{1}{2}\left[\left(\lambda_{1}^{2}+\lambda_{2}^{2}\right)-\left(\lambda_{1}+\lambda_{2}\right)\right]=\frac{1}{2}\left(t^{2}-2 d-t\right) .
$$

On the other hand,

$$
\frac{1}{2}\left(t^{2}-2 d-t\right) \geq \frac{1}{4}\left(t^{2}-4 d\right) \geq 0
$$

for all $(t, d)$ such that $t \neq 1$. For $t=1, t^{2}-4 d \geq 0$ implies $d \leq 1 / 4$ or equivalently, as $d$ is an integer, $d \leq 0$. Then $b_{2}=(1 / 2)\left(t^{2}-2 d-t\right)=-d \geq 0$. Finally, for all pairs $(t, d)$ we have that $b_{2} \geq 0$. What is more, we know by Theorem 4.1(c) that if $(t, d) \notin E_{e}$, then $b_{2} \neq 0$.

Now we show that $b_{4} \geq 0$, and then by Theorem 4.1(b) we will get the desired conclusion. We have:

$$
\begin{aligned}
4 b_{4}=L\left(f^{4}\right)-L\left(f^{2}\right) & =\left(\lambda_{1}^{4}+\lambda_{2}^{4}\right)-\left(\lambda_{1}^{2}+\lambda_{2}^{2}\right) \\
& =\left(t^{2}-2 d\right)^{2}-2 d^{2}-\left(t^{2}-2 d\right)=2 d^{2}+\left(2-4 t^{2}\right) d+t^{4}-t^{2} .
\end{aligned}
$$

It is non-negative for $d$ satisfying:

$$
d \leq \frac{1}{2}\left(2 t^{2}-1-\sqrt{2 t^{4}-2 t^{2}+1}\right) .
$$


On the other hand, by the assumption $d \leq t^{2} / 4$. Thus, if $t$ is such that $t^{2} / 4 \leq$ $(1 / 2)\left(2 t^{2}-1-\sqrt{2 t^{4}-2 t^{2}+1}\right)$, then $b_{4}$ is non-negative. The last inequality is satisfied for $|t|>1$. For $|t| \leq 1, d<0$ implies that (5.2) is satisfied. As a result, we must only check whether $b_{4} \geq 0$ for $(t, d)$ such that $|t| \leq 1$ and $0 \leq d \leq t^{2} / 4$, but then we get that $(t, d) \in E_{e}$ and so $b_{4}=0$. This ends the proof.

\section{REFERENCES}

[1] I. K. Babenko And S. A. Bogatyi, The behavior of the index of periodic points under iterations of a mapping, Math. USSR Izv. 38 (1992), 1-26.

[2] R. F. Brown, The Lefschetz Fixed Point Theorem, Glenview, New York, 1971.

[3] S. N. Chow, J. Mallet-Parret and J. A. Yorke, A periodic point index which is a bifurcation invariant, Geometric Dynamics (Rio de Janeiro, 1981), Springer Lecture Notes in Math., vol. 1007, Berlin, 1983, pp. 109-131.

[4] A. Dold, Fixed point indices of iterated maps, Invent. Math. 74 (1983), 419-435.

[5] G. Graff and J. Jezierski, Minimal number of periodic points for $C^{1}$ self-maps of compact simply-connected manifolds, Forum Math. (to appear).

[6] G. Graff and J. Jezierski, Minimal number of periodic points for smooth self-maps of $S^{3}$, preprint.

[7] G. Graff and P. Nowak-Przygodzki, Fixed point indices of iterations of $C^{1}$ maps in $\mathbb{R}^{3}$, Discrete Contin. Dynam. Systems 16 (2006), 843-856.

[8] M. Greenberg, Lectures on Algebraic Topology, Benjamin, Menlo Park, CA, 1971.

[9] A. Guillamon, X. Jarque, J. Llibre, J. Ortega and J. Torregrosa, Periods for transversal maps via Lefschetz numbers for periodic points, Trans. Amer. Math. Soc. 347 (1995), 4779-4806.

[10] J. Jezienski, Wecken's theorem for periodic points in dimension at least 3, Topology Appl. 153 (2006), 1825-1837.

[11] B. J. Jiang, Lectures on the Nielsen Fixed Point Theory, Contemp. Math., vol. 14, Amer. Math. Soc., Providence, 1983.

[12] J. Jezierski and W. Marzantowicz, Homotopy methods in topological fixed and periodic points theory, Topological Fixed Point Theory and Its Applications, 3., Springer, Dordrecht, 2005.

[13] W. Marzantowicz and P. Przygodzki, Finding periodic points of a map by use of a k-adic expansion, Discrete Contin. Dynam. Systems 5 (1999), 495-514.

[14] M. Shub and P. Sullivan, A remark on the Lefschetz fixed point formula for differentiable maps, Topology 13 (1974), 189-191.

Grzegorz GrafF

Faculty of Applied Physics and Mathematics

Gdańsk University of Technology

Narutowicza $11 / 12$

80-952 Gdańsk, POLAND

E-mail address: graff@mif.pg.gda.pl

TMNA : Volume $33-2009-\mathrm{N}^{\circ} 1$ 